\title{
The effect of nilvadipine on bloodflow in the dorsal pedis artery in type 2 diabetic patients - a study using duplex Doppler ultrasonography
}

\author{
Hirohito Sone, Yukichi Okuda, Masakazu Mizutani, Yasushi Kawakami, Chieko Bannai, \\ Kamejiro Yamashita
}

\begin{abstract}
Summary
The effects of nilvadipine on the peripheral circulation in the lower extremities using a duplex system of twodimensional colour and pulse Doppler ultrasonography were studied in 32 patients with type 2 diabetes mellitus and mild essential hypertension. The patients (19 men and 13 women) were randomly divided into treatment and control groups. The anatomical cross-sectional area and blood flow index of the dorsal pedis artery were determined by colour and pulse Doppler ultrasonography before and $60 \mathrm{~min}$ after administration of $4 \mathrm{mg}$ nilvadipine or placebo. Pulse rate and blood pressure were measured simultaneously. There were no significant changes in pulse rate or blood pressure after administration of either drug. Both cross-sectional areas (from $4.3 \pm 0.4$ to $\left.5.2 \pm 0.5 \mathrm{~mm}^{2}, p<0.05\right)$ and blood flow index (from $40.3 \pm 4.3$ to $58.8 \pm 9.0$, $p<0.05)$ were significantly increased in the treatment group, whereas there were no significant changes in either measurement in the control group. The findings showed that a single administration of nilvadipine increases blood flow in the dorsal pedis arteries of diabetic patients.
\end{abstract}

Keywords: nilvadipine, Doppler flowmetry, diabetes mellitus

Nilvadipine (5-isopropyl-3-methyl-2-cyano-1, 4-dehydro-6-methyl-4-(m-nitrophenyl)-3, 5pyridinedicarboxylate), a dihydropyridinederivative, like nifedipine and nicardipine, is a calcium-channel blocker which was first synthesized in Japan. It has a specific dilational effect on cerebral ${ }^{1-4}$ and coronary ${ }^{1,5}$ arteries and is already widely used as a hypotensor and cerebral circulation ameliorant.

Clinical application of recently developed, high-frequency probes for colour and pulse Doppler ultrasonography has made it possible to observe blood flow in superficial vessels and peripheral haemodynamics in the lower extremities under physiologic conditions. Circulatory disturbances of the lower extremities in patients with type 2 diabetes mellitus, such as diabetic gangrene and arteriosclerosis oblit- erans, are still problems awaiting a solution. We examined the effect of nilvadipine on peripheral circulation in the lower extremities using a new system of colour and pulse Doppler ultrasonography in type 2 diabetic patients with mild essential hypertension.

\section{Patients and methods}

The subjects were 32 out-patients with type 2 diabetes at Tsukuba University Hospital (19 men and 13 women) with either a systolic blood pressure of more than $150 \mathrm{mmHg}$ or a diastolic blood pressure of more than $90 \mathrm{mmHg}$ without antihypertensive therapy or any other medication except insulin (four patients) or oral hypoglycaemics (glibenclamide or gliclazide, fifteen patients). All patients were asymptomatic or had symptoms of only numbness or coldness (the first degree of Fontaine's Classification) in their legs and all of their pulses of both dorsal pedis arteries were palpable. None of the patients had calcification of the aorta or arteries of the lower extremities by radiographic observation, and none of their ankle pressure indices was 0.9 or less. Sixteen patients were randomly assigned to the nilvadipine group by the sealed envelope method and were given one tablet of nilvadipine $(4 \mathrm{mg})$. The other 16 were assigned to the control group and were given one tablet of placebo. Table 1 shows the characteristics of the patients in each group. There were no significant differences between these groups in age, sex, stage of diabetes mellitus, or severity of diabetic complications. All patients gave informed consent to participate in the study, which was conducted according to the guidelines expressed in the Declaration of Helsinki and was also approved by the Ethical Research Committee of the University of Tsukuba.

\begin{tabular}{|l|}
\hline Properties of nilvadipine \\
\hline - dihydropyridine-derivative calcium-channel \\
blocker used as a antihypertensor and cerebral \\
circulation ameliorant in Japan \\
- has a great vascular selectivity and causes \\
pronounced vasodilatation without \\
cardiodepressant effects because of its high \\
affinity for vascular calcium channels \\
\hline
\end{tabular}

Box 1 
Table 1 Patient characteristics of each group

\begin{tabular}{lll}
\hline & Nilvadipine & Control \\
\hline No. of cases (men/women) & $10 / 6$ & $9 / 7$ \\
Age (years) & $62.5 \pm 9.6$ & $59.2 \pm 11.5$ \\
Body mass index $\left(\mathrm{kg} / \mathrm{m}^{2}\right)$ & $26.8 \pm 3.9$ & $26.6 \pm 5.9$ \\
HbA 1 ( $)$ & $8.1 \pm 2.5$ & $8.0 \pm 1.4$ \\
Treatment of diabetes & & \\
$\quad$ Diet & 6 & 7 \\
OHA & 8 & 7 \\
Insulin & 2 & 2 \\
Retinopathy & & \\
$\quad$ absent & 7 & 5 \\
preproliferative & 7 & 7 \\
proliferative & 2 & 4 \\
Nephropathy & & 8 \\
$\quad$ absent & 9 & 6 \\
early & 5 & 2 \\
$\quad$ overt & 2 & 10 \\
Neuropathy & & 4 \\
absent & 8 & 2 \\
mild & 6 & \\
severe & 2 & \\
\hline
\end{tabular}

Results shown as mean $\pm \mathrm{SD}$; $\mathrm{HbA}_{1 \mathrm{C}}$ : glycohaemoglobin $\mathrm{A}_{1 \mathrm{C}}$; OHA: oral hypoglycaemic agents.

The ultrasonic duplex system (SSA-270A, Toshiba Co, Yokohama) equipped with a twodimensional colour and pulse Doppler function was used with a linear electron probe at a frequency of $7.5 \mathrm{mHz}$, which permits detection of flow at a minimum rate of about $1 \mathrm{~cm} / \mathrm{s}$. Blood flow images were observed according to the flow rate and the rate-variance modes. The frequency for the pulse Doppler method was also $7.5 \mathrm{mHz}$, and the pulse repetition frequency was set at $3-6 \mathrm{kHz}$. The sampling width for measurement of blood flow rate was set at 1-2 mm, and frequency analysis of the pulse Doppler was made by fast Fourier transformation while making angular compensation of 70 degrees or less. The anatomical crosssectional area (CSA) was determined from the internal diameter of the sagittal section of the artery in its systolic phase. The blood flow index (BFI) was determined from the product of the mean value of a single beat of instantaneous maximum flow velocity and the CSA of the dorsal pedis artery (figure 1). In practice, sample volumes for the pulse Doppler method were set in the dorsal pedis artery, and the

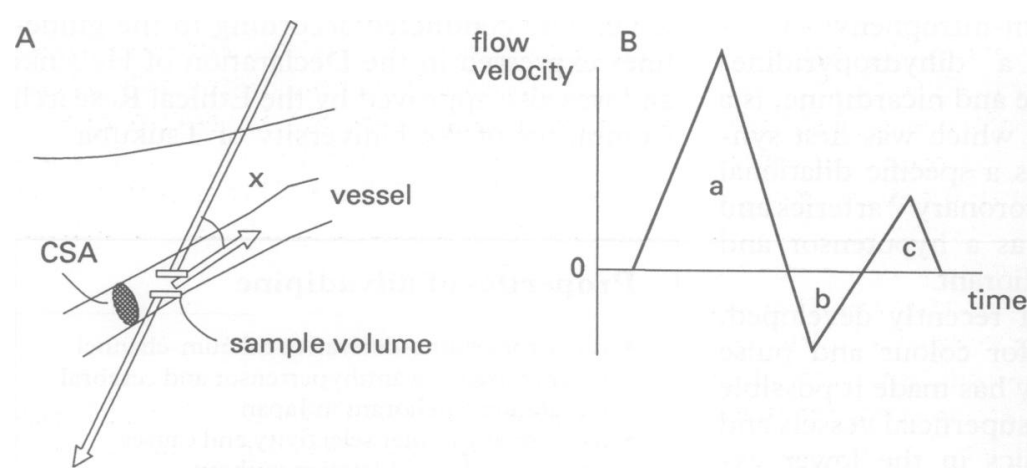

Figure 1 Method for determination of the blood flow index (BFI). (A) Twodimensional image of the colour Doppler mode. CSA: cross-sectional area of the vessel; X: angle for compensation. (B) The waveform of the pulse Doppler mode. $\mathrm{BFI}=\mathrm{CSA} \times\{(\mathrm{a}+\mathrm{c})-\mathrm{b}\}$. blood flow at this site was directly determined, while sagittal and longitudinal sections of this artery were visualised in real time on B-mode tomograms. The position and compensated angle of the sample volume were recorded on an instant photograph, and the position of the probe was recorded on the skin, to ensure the reproducibility of the position of the probe and conditions before and after administration.

Before receiving medication, the patients rested in a supine position for $20 \mathrm{~min}$ at a room temperature of $25^{\circ} \mathrm{C}$. The CSA and BFI were determined at a proximal point along the right dorsal pedis artery immediately before and $60 \mathrm{~min}$ after administration of each drug. Blood pressure of the upper arm and the pulse rate were measured simultaneously during ultrasonography. All parameters were measured twice at the same site, and mean values were obtained. Each value was expressed as mean \pm standard error. The unpaired $t$-test was used for statistical analysis of results, and values of $p<0.05$ were considered significant.

\section{Results}

As shown in table 2, there was no significant difference in blood pressure between the two groups, although it tended to decrease in the nilvadipine group (from $158.3 \pm 4.3 / 85.4 \pm 2.4$ $\mathrm{mmHg}$ before administration to $147.1 \pm 6.0$ / $83.4 \pm 3.5 \mathrm{mmHg} 60 \mathrm{~min}$ after administration). There was no significant change in pulse rate after administration in either group. The CSA significantly increased from $4.3 \pm 0.4$ $\mathrm{mm}^{2}$ to $5.2 \pm 0.5 \mathrm{~mm}^{2}$ (p < 0.05) after administration of nilvadipine, and the BFI also in-

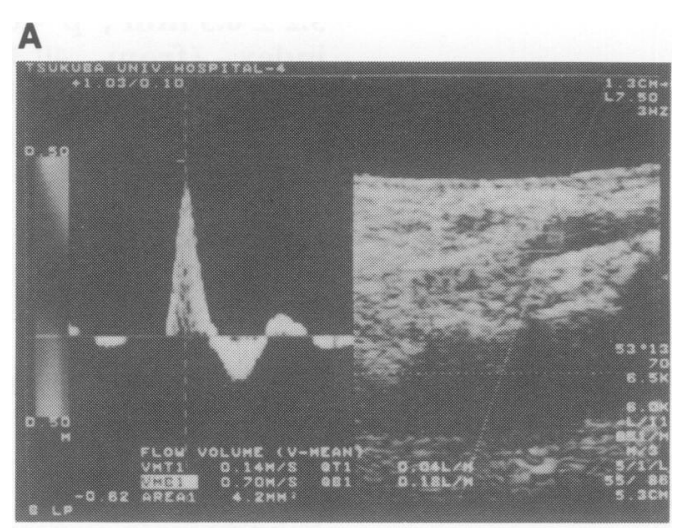

B

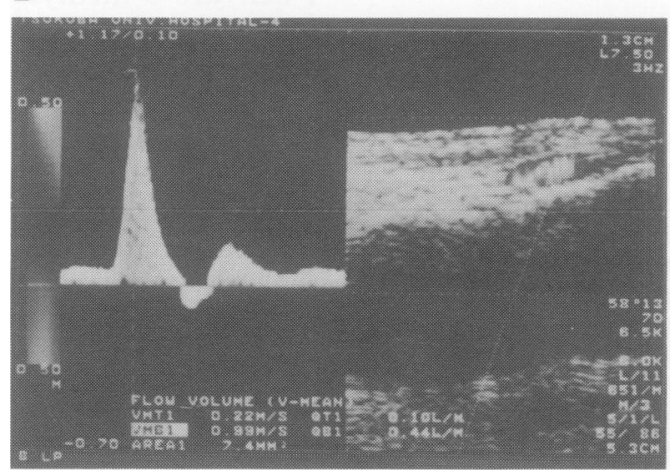

Figure 2 An example of the Doppler image obtained before (A) and after (B) the administration. In this patient, CSA increases from 4.2 to $7.4 \mathrm{~mm}^{2}$. 
Table 2 Haemodynamic changes before and $60 \mathrm{~min}$ after oral administration of each drug

\begin{tabular}{|c|c|c|c|c|}
\hline & \multicolumn{2}{|l|}{ Nilvadipine } & \multicolumn{2}{|l|}{ Control } \\
\hline & $0 \min$ & $60 \mathrm{~min}$ & $0 \min$ & $60 \min$ \\
\hline Pulse rate (beats/min) & $71.9 \pm 2.6$ & $69.5 \pm 4.3$ & $67.0 \pm 3.1$ & $66.3 \pm 3.1$ \\
\hline \multicolumn{5}{|l|}{ Blood pressure (mmHg) } \\
\hline systolic & $158.3 \pm 4.3$ & $147.1 \pm 6.0$ & $146.4 \pm 4.3$ & $148.0 \pm 5.1$ \\
\hline diastolic & $85.4 \pm 2.4$ & $83.4 \pm 3.5$ & $81.4 \pm 3.1$ & $79.7 \pm 1.9$ \\
\hline Cross-sectional area $\left(\mathrm{mm}^{2}\right)$ & $4.3 \pm 0.4$ & $5.2 \pm 0.5^{\star}$ & $4.0 \pm 0.5$ & $3.8 \pm 0.8$ \\
\hline Blood flow index & $40.3 \pm 4.3$ & $58.8 \pm 9.0^{\star}$ & $35.9 \pm 6.5$ & $32.5 \pm 6.6$ \\
\hline
\end{tabular}

Results shown as mean $\pm \mathrm{SE} ;{ }^{\star} \mathrm{p}<0.05$

creased from $40.3 \pm 4.3$ to $58.8 \pm 9.0$ ( $\mathrm{p}<$ 0.05 ). There was no significant change in any of the measurements in the control group. None of the patients showed any side effects. Figure 2 shows an example of the Doppler image before and after administration.

\section{Discussion}

The incidence of peripheral arterial lesions in diabetic patients is about five times as high as that in non-diabetics, and about $30 \%$ of diabetic patients have such lesions. ${ }^{6}$ These peripheral vascular disorders cause diabetic gangrene and aggravate diabetic neuropathy through intraneural, epineural and adventitial ischaemia, ${ }^{7}$ in addition to various metabolic disorders. ${ }^{8}$ Diabetic neuropathy itself also causes peripheral circulatory disturbances. Early detection and evaluation of any decrease in peripheral blood flow in diabetic patients is therefore important. There is a concomitant need for drugs with a peripheral vasodilational effect.

Thanks to recent advances in high-frequency probes, it is now possible to evaluate low flow rates of peripheral vessels accurately and non-invasively in clinical settings. In contrast to indirect methods involving conventional thermography or even to recent laser Doppler flow meters, ${ }^{9}$ the present method allows close observation of peripheral arterial dilatation and blood flow volume during realtime examination of blood flow. Therefore, it is expected to be clinically applicable in a variety of settings. We are now considering the application of this approach for the comparison of the acute actions of a variety of different vasodilators and the method may offer a direct and objective index of vasodilator activity.

The vasodilatory effect of nilvadipine is more marked than its inhibitory effect on the myocardium, and the results of a previous study in dogs ${ }^{1}$ also showed a dose-dependent increase in blood flow. Nilvadipine produced the greatest increase in blood flow in the coronary artery, followed by the vertebral artery, mesenteric artery, and femoral artery. A study of its effect on renal blood flow in patients with essential hypertension failed to show any significant changes, in spite of decreases in renal perfusion pressure and renal vascular resistance. ${ }^{10} \mathrm{~A}$

1 Ohtsuka M, Ono T, Hiroi J, Etsumi K, Kikuchi H, Kumada S. Comparison of the cardiovascular effect of FR34235, a S. Comparison of the cardiovascular effect of FR34235, a
new dihydropyridine, with other calcium antagonists. $\mathcal{f}$ Cardiovasc Pharmacol 1983; 5: 1074-82.

\begin{tabular}{|l|}
\hline Summary points \\
\hline - the effect of nilvadipine on the dorsal pedis \\
artery flow in a group of type 2 diabetic \\
patients was determined by duplex Doppler \\
ultrasonography \\
- significant increase of both cross-sectional \\
area and blood flow was observed in the \\
treatment group
\end{tabular}

Box 2

study in the canine femoral artery demonstrated a $10-15 \%$ increase in blood flow ${ }^{1}$ at a blood level of $0.5-0.7 \mathrm{ng} / \mathrm{ml}$, the effective blood level in humans. ${ }^{11}$ However, there have been no studies in humans or at locations more peripheral than the femoral artery. The present findings confirmed that nilvadipine directly dilates the dorsal pedis artery by an average $21 \%$ and increases blood flow volume in the peripheral arteries of the lower extremities by $45 \%$ in patients with type 2 diabeies associated with mild hypertension.

Although blood pressure tended to decrease after administration of nilvadipine, the change was not significant. The absence of a significant difference may be because the values were determined only $1 \mathrm{~h}$ after a single dose. There was no significant change in pulse rate. This suggests that the observed increase in blood flow was mainly dependent on a decrease in vascular resistance due to peripheral vasodilatation. Pharmacokinetically, the drug reached its maximum blood level $1.1 \mathrm{~h}$ after a single dose, and its half-life time was about $11 \mathrm{~h}^{12}$ Therefore, the increase in blood flow produced by the drug may continue for more than $1 \mathrm{~h}$, although we performed only a single measurement $1 \mathrm{~h}$ after administration. However, this is a single-dose study demonstrating a class effect of calcium antagonists and long-term data are required before this agent can be concluded to be effective for the failure of lower limb circulation, such as diabetic gangrene or arteriosclerosis obliterans.

Thanks are due to Miss Motomi Ezure and Miss Yoko Yokokura for their technical and secretarial assistance.

2 Tagawa K, Iino K. The effects of nilvadipine (FK235) on the cerebral circulation. Rinsho Kenkyu ( $\mathfrak{f p n}_{\mathrm{f}} \boldsymbol{f}$ Clin Exp Med) 1991; 68: 293-300. (Japanese). 
3 Kobayashi S, Yamaguchi S, Okada K, Suyama N, Murao M, Kakimatsu T. Effect of nilvadipine on cerebral circulation. Jpn Pharmacol Ther 1992; 20: 603-10. (Japanese).

4 Furuichi Y, Takakura S, Satoh H, Mori J, Kosaka M. The effect of nilvadipine, a dihydropyridine type calcium channel blocker, on local cerebral blood flow in rats. fpn $\mathcal{Y}$ Pharmacol 1992; 58: 457-60.

5 Warltier DC, Zyvoloski MG, Brooks HL, Gross GJ.

Warltier DC, Zyvoloski MG, Brooks HL, Gross GJ. Beneficial effects of the dihydropyridine calcium antagonist,
FR34235, in a chronic coronary occlusion model. Eur $₹$ Clin Pharmacol 1980; 18: 395-8.

6 Coffman JD. Intermittent claudication - be conservative. $N$ Engl f Med 1991; 325: 577-8.

7 Dyck PJ, Doll SC, Cromey DC. Pathogenesis of diabetic neuropathy. Ann Neurol 1986; 19: 450-7.

8 Greene DA, Lattimer SA, Sima AAF. Sorbitol, phosphoinositides and sodium-potasium-ATPase in the pathogenesis of diabetic complications. $N$ Engl f Med 1987; 316: 599-606.
9 Nilsson GE. A new instrument of continuous measurement of tissue blood flow by light beating spectroscopy. IEEE Trans Biomed Eng 1980; 27: 12-9.

10 Takahana $T$, Ohta $H$, Yamamoto $Y$, et al. Renal function during antihypertensive therapy with a calcium antagonist, nilvadipine. Yakuri Chiryo (Basic Pharmacol Ther) 1989; 37: 2155-60. (Japanese).

11 Umeda $T$ Naomi $S$, Iwaoka $D$, et al. The antihypertensive effect and pharmacokinetics of nilvadipine (FK235) in essential hypertension. $f p n f$ Clin Pharmacol Ther 1986; 17: 735-48. (Japanese, abstract in English)

12 Terakawa M, Tokuma Y, Shishido A, Noguchi H. Pharmacokinetics of nilvadipine in healthy volunteers. $f$ Clin Pharmacol 1987; 27: 111-7. 